\title{
PULSATILE FLOW IN CIRCULAR TUBES OF VARYING CROSS-SECTION WITH SUCTION/INJECTION
}

\author{
PEEYUSH CHANDRA ${ }^{1}$ and J. S. V. R. KRISHNA PRASAD ${ }^{1}$
}

(Received 21 May 1991; revised 31 July 1992)

\begin{abstract}
We consider here pulsatile flow in circular tubes of varying cross-section with permeable walls. The fluid exchange across the wall is accounted for by prescribing the normal velocity of the fluid at the wall. A perturbation analysis has been carried out for low Reynolds number flows and for small amplitudes of oscillation. It has been observed that the magnitude of the wall shear stress and the pressure drop decrease as the suction velocity increases. Further, as the Reynolds number is increased, the magnitude of wall shear stress increases in the convergent portion and decreases in the divergent portion of a constricted tube.
\end{abstract}

\section{Introduction}

The study of pulsatile flow in a circular tube has attracted the researchers for a long time due to its importance in understanding the fluid mechanical aspects of blood flow. Since the studies of Womersley $[10,11]$ who considered oscillatory flow in cylindrical tube of uniform cross-section, many research workers have contributed to the study of this complex flow situation by considering its different aspects. For example, in order to account for the non-uniform lumen of blood vessels, Rao and Rathna Devanathan [7] and Schneck and Ostrach [8] studied pulsatile flow through circular tubes of varying cross-section at low Reynolds number. Bitoun and Bellet [1] analysed pulsatile flow with reference to stenosis in microcirculation. In these studies the tube wall is taken to be impermeable. However, in the case of small blood vessels, the permeability of the walls

\footnotetext{
'Department of Mathematics, Indian Institute of Technology, Kanpur, 208016, India

(C) Australian Mathematical Society, 1994, Serial-fee code 0334-2700/94
} 
becomes important. Moreover, the experimental investigations have revealed that the pulsatile nature of the blood flow persists in the small blood vessels also $[2,9]$. In view of this, we consider, here, pulsatile blood flow in circular tubes of varying cross-section with permeable walls. It may be remarked that the steady flow of a viscous fluid through a circular tube with a permeable wall has been studied by Macey $[3,4]$ with reference to the flow in proximal renal tubules. This study was later extended to the flow through circular tubes of varying cross-section and permeable wall by Radhakrishnamacharya et al. [6].

The physiological situation of blood flow is highly complex. Therefore, to study the effect of tube wall permeability on pulsatile flow, we make some simplifying assumptions. In this analysis, we assume that blood is a Newtonian fluid and that the blood vessel is a straight, rigid circular tube of varying crosssection. Further, the permeability of the wall is considered by prescribing the normal velocity of the fluid at the wall. This suction/injection velocity of the fluid is taken to be pulsatile. The analyis is restricted to low Reynolds number flows.

\section{Formulation of the problem}

The pulsatile motion of a Newtonian incompressible fluid in an axisymmetric circular tube with permeable walls and varying cross-section is considered. The radius of the tube varies slowly along the axial direction, i.e., if we employ cylindrical polar co-ordinates $(X, R, \theta)$ such that $R=0$ is the axis of symmetry for the tube, then the tube radius $R=A(X)$ is given as [5]

$$
A(X)=A_{0} S\left(\varepsilon X / A_{0}\right) \text { with } S(0)=1,
$$

where $\varepsilon=\left(A_{0} / L\right) \ll 1$ is tube wall slope parameter, $A_{0}$ is tube radius at $X=0$ and $L$ is the characteristic length of the tube. It can be noted that $\varepsilon=0$ gives the case of tube with uniform radius. The equations of motion governing the axisymmetric flow are:

$$
\begin{aligned}
\frac{\partial U}{\partial T}+U \frac{\partial U}{\partial X}+V \frac{\partial U}{\partial R} & =-\frac{1}{\rho} \frac{\partial P}{\partial X}+v\left[\frac{\partial^{2} U}{\partial X^{2}}+\frac{1}{R} \frac{\partial}{\partial R}\left(R \frac{\partial U}{\partial R}\right)\right] \\
\frac{\partial V}{\partial T}+U \frac{\partial V}{\partial X}+V \frac{\partial V}{\partial R} & =-\frac{1}{\rho} \frac{\partial P}{\partial X}+v\left[\frac{\partial^{2} V}{\partial X^{2}}+\frac{1}{R} \frac{\partial}{\partial R}\left(R \frac{\partial U}{\partial R}\right)-\frac{V}{R^{2}}\right] \\
\frac{\partial U}{\partial X}+\frac{1}{R} \frac{\partial}{\partial R}(R V) & =0
\end{aligned}
$$


where $(U, V, 0)$ are velocity components in $(X, R, \theta)$ directions, $T$ is the time variable, $P$ is the pressure, $v$ is the kinematic coefficient of viscosity and $\rho$ is the constant fluid density.

To consider the permeability effect of the tube wall, we prescribe the suction/injection velocity of the fluid at the wall which is assumed to consist of a steady part and an oscillatory part. Thus, the normal component of the fluid velocity at the tube wall is given by:

$$
V-\frac{d A}{d X} U=V_{s}\left(1+\delta e^{i n T}\right)\left[1+\left(\frac{d A}{d X}\right)^{2}\right]^{1 / 2} \quad \text { at } \quad R=A(X),
$$

where $V_{s}$ is the steady state suction/injection velocity and $\delta$ is the ratio of the amplitudes of the oscillatory and steady parts of the suction/injection velocity and $n$ is the frequency of oscillation.

The no slip condition implies that the tangential velocity is zero at the wall i.e.

$$
U+\frac{d A}{d X} V=0 \quad \text { at } \quad R=A(X) .
$$

The axisymmetry of the flow gives

$$
\frac{\partial U}{\partial R}=0 \quad \text { and } \quad V=0 \quad \text { at } \quad R=0 .
$$

Further, the flux at the initial cross-section (i.e. $X=0$ ) is assumed to be in phase with the suction/injection velocity and is prescribed as

$$
Q=Q_{s}\left(1+\delta e^{i n T}\right) \quad \text { at } \quad X=0,
$$

where $Q_{s}$ is the steady state flux at the initial cross-section.

\section{Analysis}

We introduce a stream function, $\Psi$, given by

$$
U=\frac{1}{R} \frac{\partial \Psi}{\partial R}, \quad V=-\frac{1}{R} \frac{\partial \Psi}{\partial X},
$$

which on elimination of the pressure from (2) and (3) gives:

$$
\frac{\partial \Omega}{\partial T}+\left[\frac{\partial \Psi}{\partial R} \frac{\partial}{\partial X}\left(\frac{\Omega}{R}\right)-\frac{\partial \Psi}{\partial X} \frac{\partial}{\partial R}\left(\frac{\Omega}{R}\right)\right]=v\left[\frac{\partial^{2} \Omega}{\partial X^{2}}+\frac{\partial}{\partial R}\left(\frac{1}{R} \frac{\partial}{\partial R}(R \Omega)\right)\right],
$$


where

$$
\Omega=\frac{\partial}{\partial R}\left(\frac{1}{R} \frac{\partial \Psi}{\partial R}\right)+\frac{\partial}{\partial X}\left(\frac{1}{R} \frac{\partial \Psi}{\partial X}\right)
$$

Further, the boundary conditions (6) and (7) in terms of $\Psi$ can be written as

$$
\begin{aligned}
& \frac{\partial \Psi}{\partial R}-\frac{d A}{d X} \frac{\partial \Psi}{\partial X}=0 \quad \text { at } \quad R=A(X) \quad \text { and } \\
& \Psi=0, \quad \frac{1}{R} \frac{\partial \Psi}{\partial X}=0, \quad \frac{\partial}{\partial R}\left(\frac{1}{R} \frac{\partial \Psi}{\partial R}\right)=0 \quad \text { as } \quad R \rightarrow 0 .
\end{aligned}
$$

The equation of continuity (4) along with the conditions (5) and (8) for axisymmetric flow gives

$$
\begin{aligned}
& \Psi=\frac{1}{2 \pi}\left(1+\delta e^{i n T}\right)\left[Q_{s}-2 \pi V_{s} \int_{0}^{X} A(\xi)\left\{1+\left(\frac{d A}{d \xi}\right)^{2}\right\}^{1 / 2} d \xi\right] \\
& \text { at } R=A(X) \text {. }
\end{aligned}
$$

Using the following non-dimensional variables

$$
\left.\begin{array}{ccc}
x=\frac{\varepsilon X}{A_{0}}, & r=\frac{R}{A_{0}}, & t=n T, \\
\phi=\frac{2 \pi \Psi}{Q_{s}}, & \omega=2 \pi A_{0}{ }^{3} \frac{\Omega}{Q_{s}}, & p=\frac{2 \pi A_{0}{ }^{3} P}{\left(\rho v Q_{s}\right)}
\end{array}\right\}
$$

and the dimensionless parameters

$$
\operatorname{Re}=\frac{Q_{s}}{\left(2 \pi v A_{0}\right)} ; \quad \alpha^{2}=\frac{n A_{0}{ }^{2}}{v} \quad \text { and } \quad v_{s}=\frac{2 \pi A_{0}{ }^{2} V_{s}}{\left(\varepsilon Q_{s}\right)}
$$

(10) and (11) and the conditions (12) and (14) can be written in non-dimensional form as:

$$
\begin{aligned}
& \alpha^{2} \frac{\partial \omega}{\partial t}+\varepsilon \operatorname{Re}\left[\frac{1}{r} \frac{\partial \phi}{\partial r} \frac{\partial \omega}{\partial x}-\frac{\partial \phi}{\partial x} \frac{\partial}{\partial r}\left(\frac{\omega}{r}\right)\right]=\varepsilon^{2} \frac{\partial^{2} \omega}{\partial x^{2}}+\frac{\partial^{2} \omega}{\partial r^{2}}+\frac{1}{r} \frac{\partial \omega}{\partial r}-\frac{\omega}{r^{2}}, \\
& \omega=\frac{1}{r}\left[\frac{\partial^{2} \phi}{\partial r^{2}}-\frac{1}{r} \frac{\partial \phi}{\partial r}+\varepsilon^{2} \frac{\partial^{2} \phi}{\partial x^{2}}\right] \\
& \frac{\partial \phi}{\partial r}+\varepsilon^{2} \frac{d S}{d x} \frac{\partial \phi}{\partial x}=0 \\
& \left.\begin{array}{l}
\partial r \quad d x \partial x \\
\phi=\left(1+\delta e^{i n t}\right)\left[1-v_{s} \int_{0}^{x} G(\xi) d \xi\right]
\end{array}\right\} \quad \text { at } r=S(x), \\
& \phi=0, \quad \frac{\partial \phi}{\partial x}=0, \quad \frac{\partial}{\partial r}\left(\frac{1}{r} \frac{\partial \phi}{\partial r}\right)=0 \quad \text { as } \quad r \rightarrow 0,
\end{aligned}
$$


where $G(x)=S(x)\left[1+\varepsilon^{2}\left(\frac{d S}{d x}\right)^{2}\right]^{\frac{1}{2}}, \operatorname{Re}$ is entrance flow Reynolds number, $\alpha^{2}$ is Womersley's parameter, and $v_{s}$ is leakage parameter.

It may be noted that $v_{s}=0$ in the boundary condition (17) means 'no leakage through the tube wall', hence it refers to the case of pulsatile flow through circular tube of varying cross-section with impermeable wall. Also $\delta=0$ in (17) would lead to the case of steady flow.

\section{Method of solution}

We assume here that the pulsatile flow consists of

(i) the steady part, and

(ii) the oscillatory part which has small amplitude of oscillation such that the terms of the order $\delta^{2}$ can be neglected, i.e. $\delta \ll 1$.

In view of this, we seek the solution of (15) to (18) in the following form:

$$
\left.\begin{array}{c}
\omega=\left(\omega_{00}+\delta e^{i t} \omega_{01}\right)+\varepsilon\left(\omega_{10}+\delta e^{i t} \omega_{11}\right)+O\left(\varepsilon^{2}, \delta^{2}\right), \\
\phi=\left(\phi_{00}+\delta e^{i t} \phi_{01}\right)+\varepsilon\left(\phi_{10}+\delta e^{i t} \phi_{11}\right)+O\left(\varepsilon^{2}, \delta^{2}\right) .
\end{array}\right\}
$$

Further, we restrict our analysis to low Reynolds number flows [5], i.e. $R e \simeq \mathrm{O}(1)$. Thus, using the perturbation scheme (19) for $\omega$ and $\phi$ in equations (15) to (18), and collecting the coefficients of $e^{i t}$ and of equal powers of $\varepsilon$, we get the following coupled equations and the corresponding boundary conditions:

(i) Zeroth order-steady part

$$
\begin{aligned}
D^{2} \omega_{00} & =0, \\
\omega_{00} & =\frac{\partial}{\partial r}\left(\frac{1}{r} \frac{\partial \phi_{00}}{\partial r}\right), \\
\frac{\partial \phi_{00}}{\partial r} & =0, \quad \phi_{00}=1-v_{s} F(x) \quad \text { at } \quad r=S(x), \\
\phi_{00} & =0, \quad \frac{\partial \phi_{00}}{\partial x}=0, \quad \frac{\partial}{\partial r}\left(\frac{1}{r} \frac{\partial \phi_{00}}{\partial r}\right)=0 \quad \text { as } \quad r \rightarrow 0,
\end{aligned}
$$

where $D^{2} \equiv \partial^{2} / \partial r^{2}+(1 / r)(\partial / \partial r)-1 / r^{2}$ and $F(x)=\int_{0}^{x} S(\xi) d \xi$.

(ii) Zeroth order-oscillatory part

$$
\begin{aligned}
D^{2} \omega_{01} & =\lambda^{2} \omega_{01}, \\
\omega_{01} & =\frac{\partial}{\partial r}\left(\frac{1}{r} \frac{\partial \phi_{01}}{\partial r}\right),
\end{aligned}
$$




$$
\begin{aligned}
& \frac{\partial \phi_{01}}{\partial r}=0, \quad \phi_{01}=1-v_{s} F(x) \quad \text { at } \quad r=S(x), \\
& \phi_{01}=0, \quad \frac{\partial \phi_{01}}{\partial x}=0, \quad \frac{\partial}{\partial r}\left(\frac{1}{r} \frac{\partial \phi_{01}}{\partial r}\right)=0 \quad \text { as } \quad r \rightarrow 0,
\end{aligned}
$$

where $\lambda^{2}=i \alpha^{2}$.

(iii) First order-steady part

$$
\begin{aligned}
D^{2} \omega_{10} & =\operatorname{Re}\left[\frac{1}{r} \frac{\partial \phi_{00}}{\partial r} \frac{\partial \omega_{00}}{\partial x}-\frac{\partial \phi_{00}}{\partial x} \frac{\partial}{\partial r}\left(\frac{\omega_{00}}{r}\right)\right], \\
\omega_{10} & =\frac{\partial}{\partial r}\left(\frac{1}{r} \frac{\partial \phi_{10}}{\partial r}\right), \\
\frac{\partial \phi_{10}}{\partial r} & =0, \quad \phi_{10}=0 \quad \text { at } \quad r=S(x), \\
\phi_{10} & =0, \quad \frac{\partial \phi_{10}}{\partial x}=0, \quad \frac{\partial}{\partial r}\left(\frac{1}{r} \frac{\partial \phi_{10}}{\partial r}\right)=0 \text { as } r \rightarrow 0 .
\end{aligned}
$$

(iv) First order-oscillatory part

$$
\begin{aligned}
& D^{2} \omega_{11}-\lambda^{2} \omega_{11}= \operatorname{Re}\left[\frac{1}{r}\left\{\frac{\partial \phi_{00}}{\partial r} \frac{\partial \omega_{01}}{\partial x}+\frac{\partial \phi_{01}}{\partial r} \frac{\partial \omega_{00}}{\partial x}\right\}\right. \\
&\left.-\left\{\frac{\partial \phi_{01}}{\partial x} \frac{\partial}{\partial r}\left(\frac{\omega_{00}}{r}\right)+\frac{\partial \phi_{00}}{\partial x} \frac{\partial}{\partial r}\left(\frac{\omega_{01}}{r}\right)\right\}\right], \\
& \omega_{11}=\frac{\partial}{\partial r}\left(\frac{1}{r} \frac{\partial \phi_{11}}{\partial r}\right), \\
& \frac{\partial \phi_{11}}{\partial r}=0, \quad \phi_{11}=0 \quad \text { at } \quad r=S(x), \\
& \phi_{11}=0, \quad \frac{\partial \phi_{11}}{\partial x}=0, \quad \frac{\partial}{\partial r}\left(\frac{1}{r} \frac{\partial \phi_{11}}{\partial r}\right)=0 \quad \text { as } \quad r \rightarrow 0 .
\end{aligned}
$$

The equations $(20 \mathrm{a}, \mathrm{b})$ and $(21 \mathrm{a}, \mathrm{b})$ are solved along with the corresponding boundary conditions to give the zeroth order $\omega$ and $\phi$ as follows

$$
\begin{aligned}
& \omega_{00}=-8\left(1-v_{s} F(x)\right) r / S^{4}, \\
& \phi_{00}=\left(1-v_{s} F(x)\right)\left(2 r^{2} S^{2}-r^{4}\right) / S^{4}, \\
& \omega_{01}=-2 \lambda\left(1-v_{s} F(x)\right) \frac{I_{1}(\lambda r)}{S^{2} I_{2}(\lambda S)}, \\
& \phi_{01}=\left(1-v_{s} F(x)\right)\left[\lambda r I_{0}(\lambda S)-2 I_{1}(\lambda r)\right] \frac{r}{\lambda S^{2} I_{2}(\lambda S)},
\end{aligned}
$$


where $I_{0}(z), I_{1}(z), I_{2}(z)$ are modified Bessel functions of order 0,1 and 2 respectively.

The expressions for the zeroth order components $\omega$ and $\phi$ (given by (24) and (27)) are substituted in (22) and (23) which are then solved for the first order components. Thus, the expressions for $\omega_{10}, \phi_{10}, \omega_{11}$ and $\phi_{11}$ are obtained in the following form:

$$
\begin{aligned}
\omega_{10}= & -2 \frac{R e}{S^{8}}\left(1-v_{s} F(x)\right)\left(g_{1}+4 g_{2}\right)\left(S^{4} r-2 S^{2} r^{3}+\frac{2}{3} r^{5}\right), \\
\phi_{10}= & \frac{1}{36} \frac{R e}{S^{8}}\left(1-v_{s} F(x)\right)\left(g_{1}+4 g_{2}\right)\left(4 S^{6} r^{2}-9 S^{4} r^{4}+6 S^{2} r^{6}-r^{8}\right), \\
\omega_{11}= & \frac{\operatorname{Re}}{\lambda^{2} S^{9} I_{2}(\lambda S)}\left(1-v_{s} F(x)\right)\left[T_{1} r I_{0}(\lambda r)-T_{2} r^{2} I_{1}(\lambda r)\right. \\
& \left.-T_{3} r^{3} I_{2}(\lambda r)+T_{4} r^{4} I_{1}(\lambda r)-8 T_{6} r-T_{7} I_{1}(\lambda r)\right] \\
\phi_{11}= & \frac{R e}{\lambda^{4} S^{9} I_{2}(\lambda s)}\left(1-v_{s} F(x)\right)\left[T_{1} r^{2} I_{2}(\lambda r)-T_{2} r^{3} I_{3}(\lambda r)\right. \\
& \left.\quad+T_{4} r^{5} I_{3}(\lambda r)-T_{5} r^{4} I_{4}(\lambda r)-\lambda^{2} T_{6} r^{4}-T_{7} I_{1}(\lambda r)-T_{8} r^{2}\right] .
\end{aligned}
$$

Here $I_{m}(z)$ is the modified Bessel function of order $m ; g_{1}, g_{2}$ and $T_{i}(i=$ 1 to 8 ) are functions of $S(x)$ and are given as follows

$$
\begin{aligned}
& g_{1}=v_{s} S(x), \quad g_{2}=\frac{1}{S}\left(1-v_{s} F(x)\right) \frac{d S}{d x} \\
& T_{1}=4 \lambda^{2} S^{5}\left[1+g_{1}+\frac{\lambda S I_{1}(\lambda S)}{I_{2}(\lambda S)} g_{2}\right] \\
& T_{2}=\lambda S^{3}\left[\left(4+\lambda^{2} S^{2}\right) g_{1}+2\left(8+\lambda^{2} S^{2}\right) g_{2}\right] \\
& T_{3}=\frac{1}{3} \lambda^{2} S^{3}\left[7 g_{1}+4\left\{3+\frac{\lambda S I_{1}(\lambda S)}{I_{2}(\lambda S)}\right\} g_{2}\right] \\
& T_{4}=\frac{1}{4} \lambda^{3} S^{3}\left(g_{1}+4 g_{2}\right), \\
& T_{5}=\frac{2}{3} \lambda^{2} S^{3}\left[5 g_{1}+2\left\{6+\frac{\lambda S I_{1}(\lambda S)}{I_{2}(\lambda S)}\right\} g_{2}\right] \\
& T_{6}=2 S^{3}\left(g_{1}+4 g_{2}\right) I_{0}(\lambda S), \\
& T_{7}=\frac{1}{\lambda I_{2}(\lambda S)}\left[T_{1}\left\{\lambda S I_{1}(\lambda S)-2 I_{2}(\lambda S)\right\}-S T_{2}\left\{\lambda S I_{2}(\lambda S)-2 I_{3}(\lambda S)\right\}\right. \\
& \left.\quad-S^{2} T_{5}\left\{\lambda S I_{3}(\lambda S)-2 I_{4}(\lambda S)\right\}+\lambda S^{4} T_{4} I_{4}(\lambda S)-2 \lambda^{2} S^{2} T_{6}\right]
\end{aligned}
$$




$$
\begin{aligned}
& T_{8}=\frac{1}{I_{2}(\lambda S)}\left[I _ { 0 } ( \lambda S ) \left\{I_{2}(\lambda S) T_{1}-S I_{3}(\lambda S) T_{2}+S^{3} I_{3}(\lambda S) T_{4}-S^{2} I_{4}(\lambda S) T_{5}\right.\right. \\
&\left.-\lambda^{2} S^{2} T_{6}\right\}-I_{1}(\lambda S)\left\{I_{1}(\lambda S) T_{1}-S I_{2}(\lambda S) T_{2}+S^{2} / \lambda\left(\lambda S I_{2}(\lambda S)\right.\right. \\
&\left.\left.\left.+2 I_{3}(\lambda S)\right) T_{4}-S^{2} I_{3}(\lambda S) T_{5}-4 \lambda S T_{6}\right\}\right] .
\end{aligned}
$$

In the above expression substituting $v_{s}=0$ gives $g_{1} \equiv 0$ which corresponds to the case of impermeable tube wall, while $g_{2} \equiv 0$ leads to the flow through tube with constant radius $(S(x) \equiv 1)$.

Shear stress at wall of tube The shear stress, $T_{w}$, at the tube wall $R=S(x)$ is defined as

$$
T_{w}=\left[\sigma_{X R}\left\{1-\left(\frac{d A}{d X}\right)^{2}\right\}+\left(\sigma_{R R}-\sigma_{X X}\right) \frac{d A}{d X}\right] /\left\{1+\left(\frac{d A}{d X}\right)^{2}\right\},
$$

where

$$
\sigma_{X R}=\mu\left[\frac{\partial U}{\partial R}+\frac{\partial V}{\partial X}\right]
$$

and

$$
\sigma_{R R}-\sigma_{X X}=-2 \mu\left[\frac{\partial U}{\partial X}-\frac{\partial V}{\partial R}\right]
$$

are calculated at $R=A(X)$.

This along with the boundary conditions at $R=S(X)$ and (9) and (11) gives the dimensionless wall shear stress, $\tau_{w}$, in the following form:

$$
\begin{aligned}
\tau_{w} & =\frac{2 \pi A_{0}^{3}}{\mu Q_{s}} T_{w} \\
& =\omega_{00}+\delta e^{i t} \omega_{01}+\varepsilon\left(\omega_{10}+\delta e^{i t} \omega_{11}\right)+\mathrm{O}\left(\varepsilon^{2}, \delta^{2}\right) \text { at } \quad R=S(X) .
\end{aligned}
$$

Thus we get from (24), (26), (28) and (30)

$$
\begin{gathered}
\tau_{w}=-\frac{8\left(1-v_{s} F(x)\right)}{S^{3}}\left[1+\delta e^{i t}\left(\frac{\lambda S I_{1}(\lambda S)}{4 I_{2}(\lambda S)}\right)-\varepsilon R e\left\{\frac{g_{1}+4 g_{2}}{12}\right.\right. \\
-\frac{\delta e^{i t}}{8 \lambda^{2} S^{6} I_{2}(\lambda S)}\left(S I_{0}(\lambda S) T_{1}-S^{2} I_{1}(\lambda S) T_{2}-S^{3} I_{2}(\lambda S) T_{3}\right. \\
\left.\left.\left.+S^{4} I_{1}(\lambda S) T_{4}-8 S T_{6}-I_{1}(\lambda S) T_{7}\right)\right\}\right]+\mathrm{O}\left(\varepsilon^{2}, \delta^{2}\right) .
\end{gathered}
$$


Pressure drop To calculate pressure, we observe that the equations of motion (2) and (3) and the non-dimensionalizing scheme (14) give the following form of dimensionless pressure, $p$,

$$
\varepsilon p=\left(p_{00}+\delta e^{i t} p_{01}\right)+\varepsilon\left(p_{10}+\delta e^{i t} p_{11}\right)+\mathrm{O}\left(\varepsilon^{2}, \delta^{2}\right)
$$

Thus the equations governing pressure components can be written as:

$$
\begin{aligned}
\frac{\partial p_{00}}{\partial x}= & \frac{1}{r} \frac{\partial}{\partial r}\left(r \omega_{00}\right) \\
\frac{\partial p_{01}}{\partial x}= & \frac{1}{r} \frac{\partial}{\partial r}\left(r \omega_{01}\right)-\frac{\lambda^{2}}{r} \frac{\partial \phi_{01}}{\partial r} \\
\frac{\partial p_{10}}{\partial x}= & \frac{1}{r} \frac{\partial}{\partial r}\left(r \omega_{10}\right)-\frac{R e}{r}\left[\frac{1}{r} \frac{\partial \phi_{00}}{\partial r} \frac{\partial^{2} \phi_{00}}{\partial r \partial x}-\omega_{00} \frac{\partial \phi_{00}}{\partial x}\right] \\
\frac{\partial p_{11}}{\partial x}= & \frac{1}{r} \frac{\partial}{\partial r}\left(r \omega_{11}\right)-\frac{\lambda^{2}}{r} \frac{\partial \phi_{11}}{\partial r} \\
& -\frac{R e}{r}\left[\frac{1}{r}\left\{\frac{\partial \phi_{00}}{\partial r} \frac{\partial^{2} \phi_{01}}{\partial r \partial x}-\frac{\partial \phi_{01}}{\partial r} \frac{\partial^{2} \phi_{00}}{\partial r \partial x}\right\}-\omega_{00} \frac{\partial \phi_{01}}{\partial x}+\omega_{01} \frac{\partial \phi_{00}}{\partial x}\right]
\end{aligned}
$$

with

$$
\frac{\partial p_{00}}{\partial r}=\frac{\partial p_{01}}{\partial r}=\frac{\partial p_{10}}{\partial r}=\frac{\partial p_{11}}{\partial r}=0 .
$$

The equation (37) shows the $p_{00}, p_{01}, p_{10}, p_{11}$ are independent of $r$, hence (33) to (36) are integrated to give the pressure drop $\Delta p(x)=p(0)-p(x)$ up to the first order as follows:

$$
\begin{aligned}
\varepsilon \Delta p=16 \int_{0}^{x} & \frac{1-v_{s} F(x)}{S^{4}} d x+2 \lambda^{2} \delta e^{i t} \int_{0}^{x} \frac{1-v_{s} F(x)}{S^{2} I_{2}(\lambda S)} I_{0}(\lambda S) d x \\
& -4 R e \varepsilon\left[\int_{0}^{x} \frac{1-v_{s} F(x)}{S^{4}}\left(3 g_{1}+4 g_{2}\right) d x\right. \\
& +2 \delta e^{i t} \int_{0}^{x}\left\{\left(1-v_{s} F(x)\right) T_{9}-\frac{4 v_{s}}{\lambda^{2} S}\left(g_{1}+4 g_{2}\right)\right\} \frac{d x}{S^{4} I_{2}(\lambda S)} \\
& +\mathrm{O}\left(\varepsilon^{2}, \delta^{2}\right)
\end{aligned}
$$

where

$$
T_{9}=2 I_{0}(\lambda S) g_{1}+\left\{2 I_{0}(\lambda S)-\lambda S I_{1}(\lambda S)+\frac{\lambda S I_{0}(\lambda S)}{I_{2}(\lambda S)}\right\} g_{2}+\frac{T_{8}}{4 \lambda^{2} S^{5}}
$$




\section{Results and discussion}

In the above analysis, the expressions of the flow variables $\phi, \omega, \tau_{w}$ and $\Delta p$ depend upon the following non-dimensional parameters

(i) $v_{s}$ leakage parameter

(ii) $R e$ : entrance Reynolds number

(iii) $\lambda=\sqrt{i \alpha^{2}}$, where $\alpha^{2}$ is Womersley's parameter, apart from the perturbation parameters $\varepsilon$ and $\delta$.

Due to the presence of complex parameter $\lambda$ these quantities appear in the complex form. This is because of the periodic boundary conditions (5) and (8) which in the non-dimensional form can be written as:

$$
\phi=\left(1+\delta e^{i \ell}\right)\left[1-v_{s} \int_{0}^{x} G(\xi) d \xi\right] \quad \text { at } \quad r=S(x)
$$

It is the real part of this condition, i.e.,

$$
\phi=(1+\delta \cos t)\left(1-v_{s} \int_{0}^{x} G(\xi) d \xi\right) \text { at } \quad r=S(x),
$$

which is of interest. Hence, for our discussion we consider only the real part of the expression of wall shear stress and the pressure drop. Thus the expressions (32) and (38) are split into real and imaginary parts and the real parts are numerically evaluated to see the effect of $v_{s}, R e$ and $\alpha$ on wall shear stress $\left(\tau_{w}\right)$ and pressure drop $(\triangle p)$. The results are obtained by taking $\varepsilon=0.1$ and $\delta=0.1$ for the following tube geometries:

(a) Locally constricted tube i.e.

$$
S(x)=\left[2-\exp \{-(x-0.5)\}^{2}\right] /\left[2-e^{-0.25}\right]
$$

(b) Sinusoidal tube

$$
S(x)=1+0.2 \sin (2 \pi x)
$$

\section{(i) Wall shear stress}

The magnitude of dimensionless wall shear stress $\left(\left|\tau_{w}\right|\right)$ is plotted in Figures 1 to 3 for locally constricted tube geometry and in Figures 4 to 6 for sinusoidal tube. In the Figures 1, 2, 4 and 5, $\left|\tau_{w}\right|$ is plotted versus $x$ (axial distance) at $t=\frac{\pi}{3}$ 
and $t=\frac{3 \pi}{4}$, while in Figures 3 and 6, its variation is shown with respect to the time parameter $(t)$ at different cross-sections $(x=0.3,0.5$ and 0.7$)$. It is clear from these figures that the values of $\left|\tau_{w}\right|$ increase in the converging portion of the tube and decrease in the diverging regions. Further, the suction of the fluid at the well reduces the flux in the tube which results in the reduction of wall shear stress. This behaviour is reflected in the figures also, e.g., the values of $\left|\tau_{w}\right|$ in the case of suction ( $v_{s}=0.2$, Figure 1 ) are less than the corresponding values of $\left|\tau_{w}\right|$ in the case of injection ( $v_{s}=-0.2$, Figure 2). The effect of the frequency parameter $(\alpha)$ on $\left|\tau_{w}\right|$ depends upon the time parameter $(t)$. The increase in $\alpha$ decreases $\left|\tau_{w}\right|$ at all the cross-sections for $t=\frac{\pi}{4}$ and $t=\frac{3 \pi}{4}$. However, this behaviour is observed only in approximately the first half of the time cycle, i.e. for $t<\pi$ and the reverse trend is seen for $t>\pi$ (Figures 3 and 6). It is noted that this variation of $\left|\tau_{w}\right|$ with respect to $\alpha$ remains similar in the case of suction as well as injection. The effect of $R e$ (Reynolds number) is to enhance wall shear stress in the converging portions and to reduce it in the diverging portions at $t=\frac{\pi}{4}$ and $t=\frac{3 \pi}{4}$. This effect if further elaborated in Figures 3 and 6. However, it is noted in the case of suction of fluid in the constricted tube that the variation of $\left|\tau_{w}\right|$ with respect to $R e$ at $x=0.3$ depends upon $t$. While $\left|\tau_{w}\right|$ increases with $\operatorname{Re}$ for $t<\pi$, it shows decreasing trend for $t>\pi$ at $x=0.3$. No such behaviour is observed in the case of injection. It is also noted that the effect of $\alpha$ and $R e$ on $\left|\tau_{w}\right|$ is not very significant in the constricted region of the tube (Figure 3, at $x=0.5$ ). In the case of sinusoidal tube (Figures 4 and 5) the effect of $R e$ is more significant and the increase/decrease of $\left|\tau_{w}\right|$ with $R e$ in converging/diverging portions is very sharp.

(ii) Pressure drop

The pressure drop $\Delta p$ is plotted versus $x$ in Figure 7 for the constricted tube and in Figure 8 for the sinusoidal tube at $t=\frac{\pi}{4}$ and $t=\frac{3 \pi}{4}$. In the case of the constricted tube $\Delta p$ at any cross-section is more in case of injection than suction. Also, in this case, pressure drops more for higher values of $R e$ and this behaviour is seen at all the cross-sections of the tube (Figure 7). However, in the case of the sinusoidal tube (Figure 8), the effect of $R e$ on $\Delta p$ is to decrease it at the initial cross-section and then to increase it for injection. In the case of suction, $\Delta p$ again decreases for the cross-sections $x>0.85$ as $R e$ increases. This shifting behaviour of $\Delta p$ with respect to $R e$ along the tube is mainly due to the converging and diverging sections of the sinusoidal tube. Moreover, pressure drop decreases with the frequency parameter $\alpha$. This behaviour is similar in both the tube geometries. 


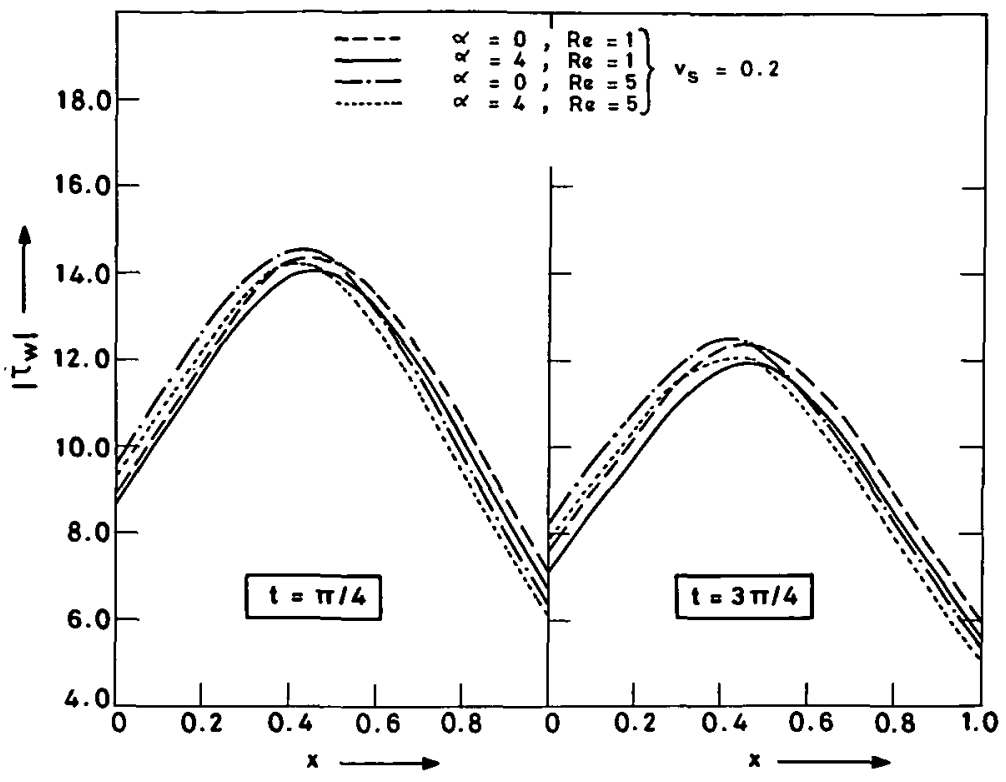

FIGURE 1. $\left|\tau_{w}\right|$ for locally constricted tube with suction at the wall.

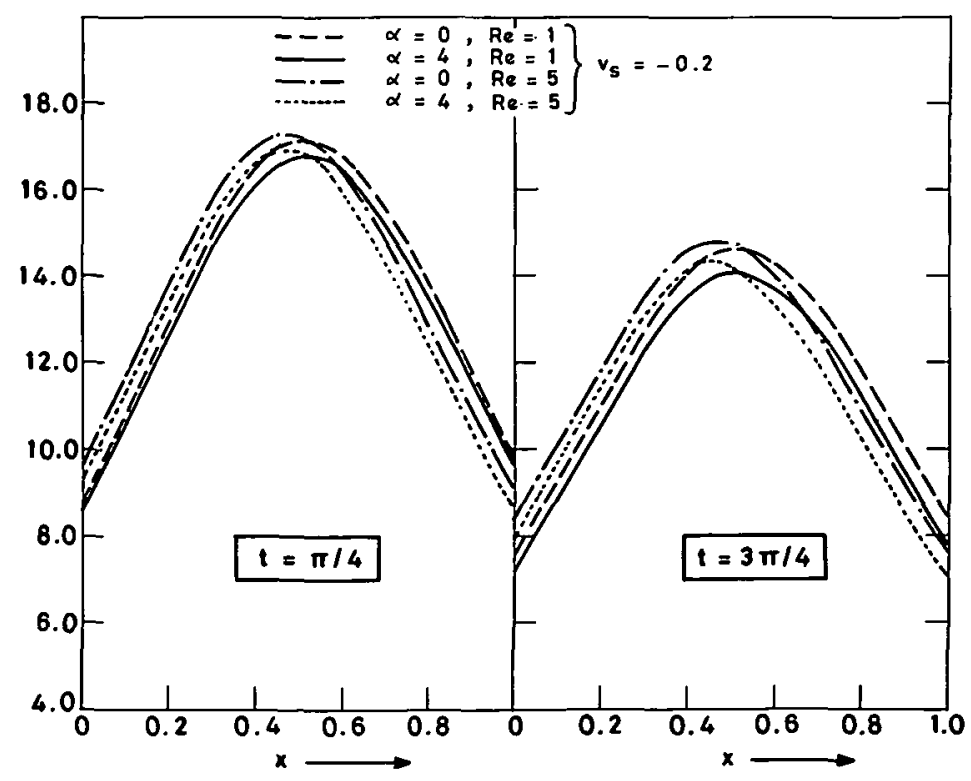

FIGURE 2. $\left|\tau_{w}\right|$ for locally constricted tube with injection at the wall. 


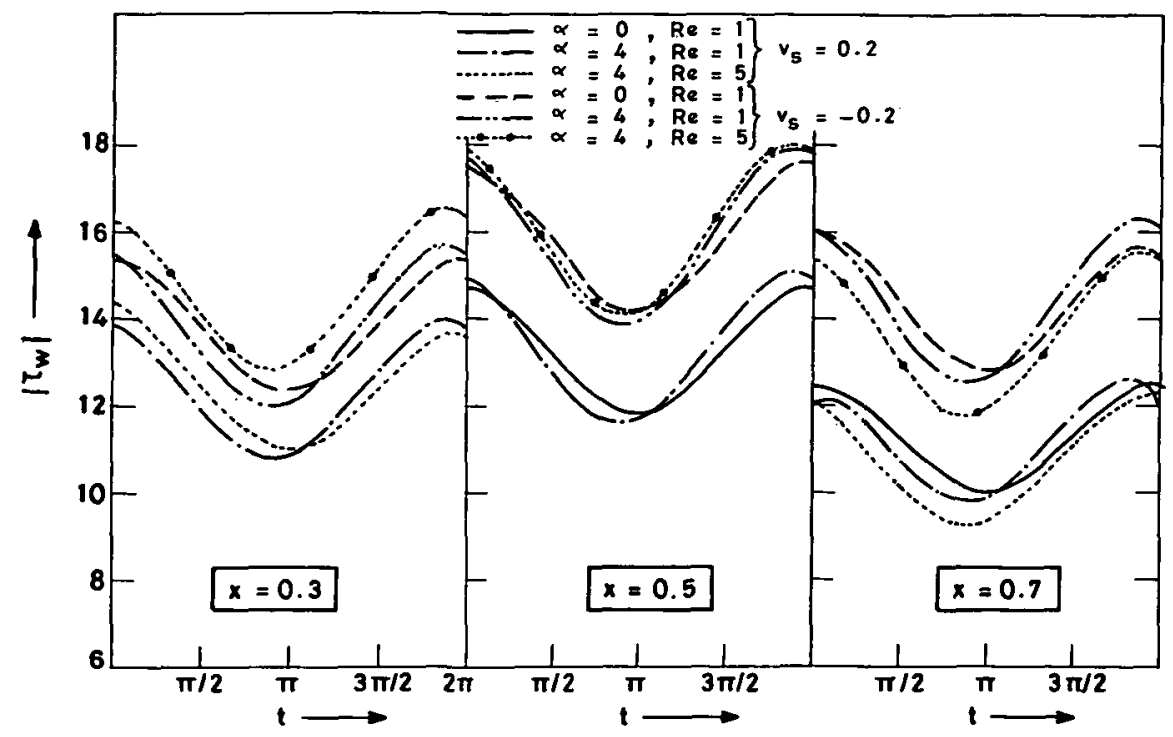

FIGURE 3. $\left|\tau_{w}\right|$ for locally constricted tube.

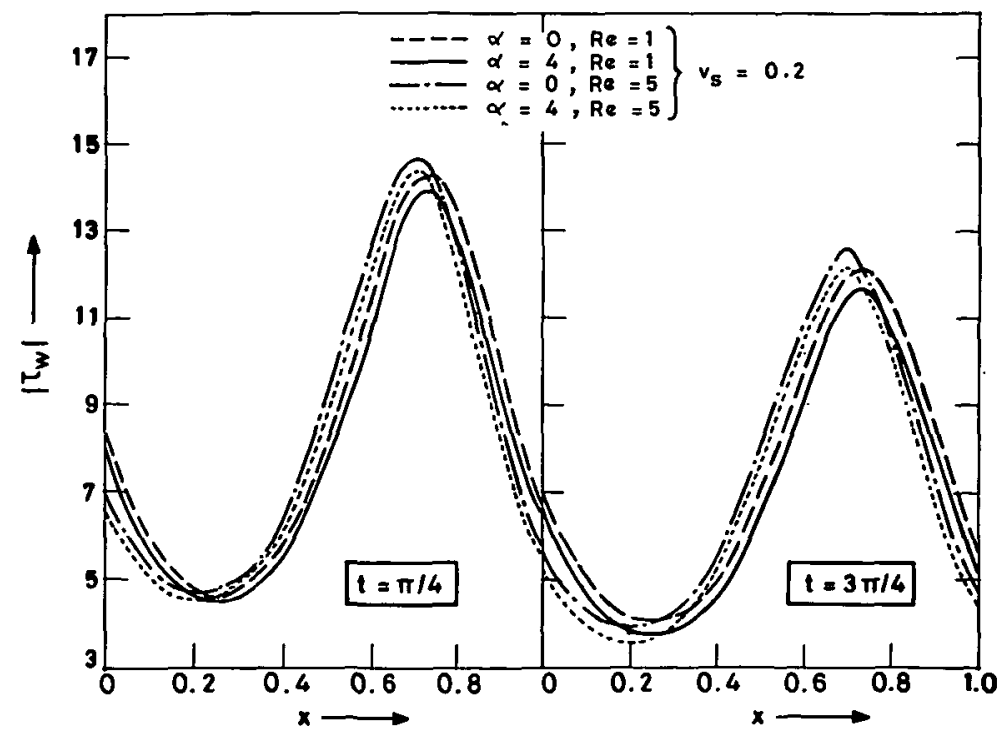

FIGURE 4. $\left|\tau_{w}\right|$ for sinusoidal tube with suction at the wall. 


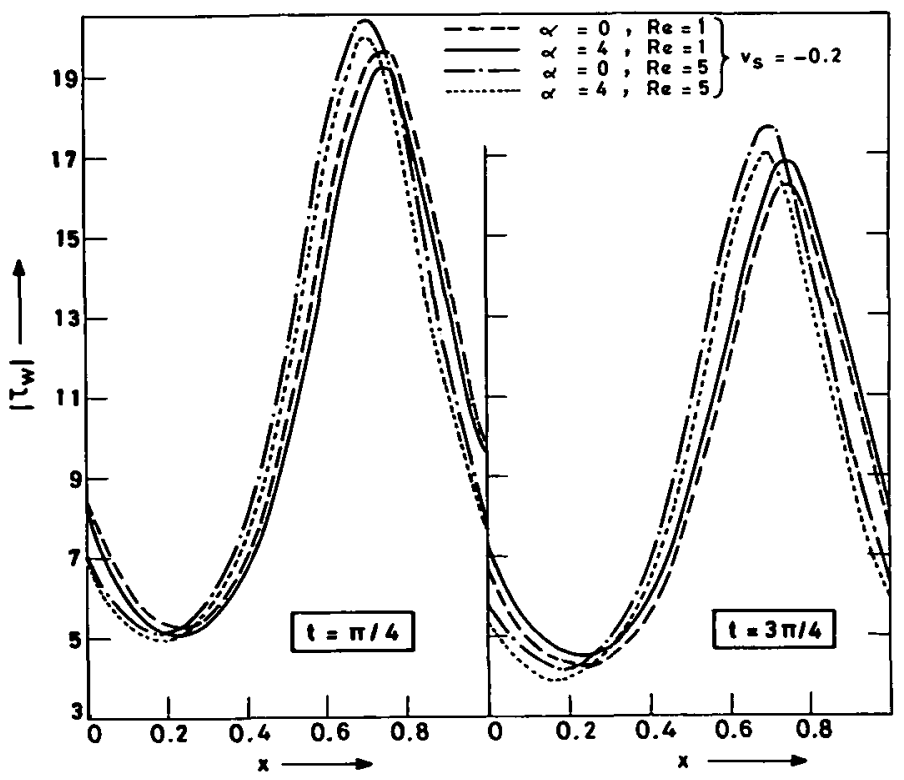

FIGURE 5. $\left|\tau_{w}\right|$ for sinusoidal tube with injection at the wall.

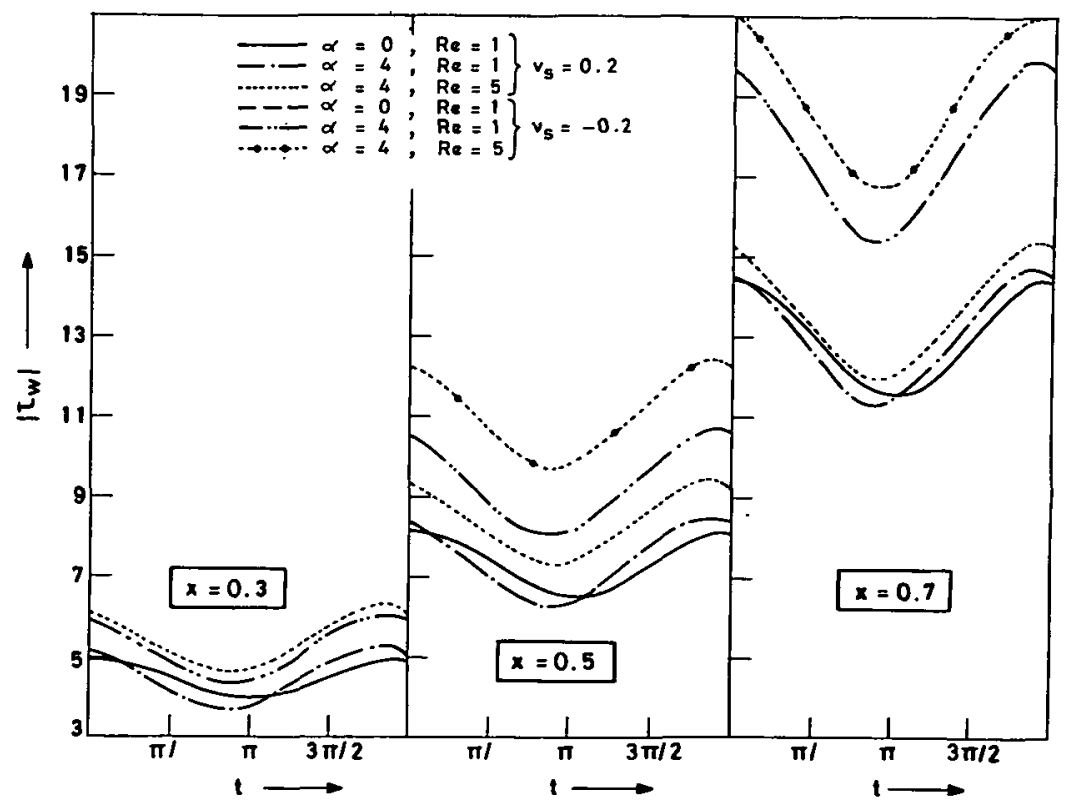

FIGURE 6. $\left|\tau_{w}\right|$ for sinusoidal tube. 


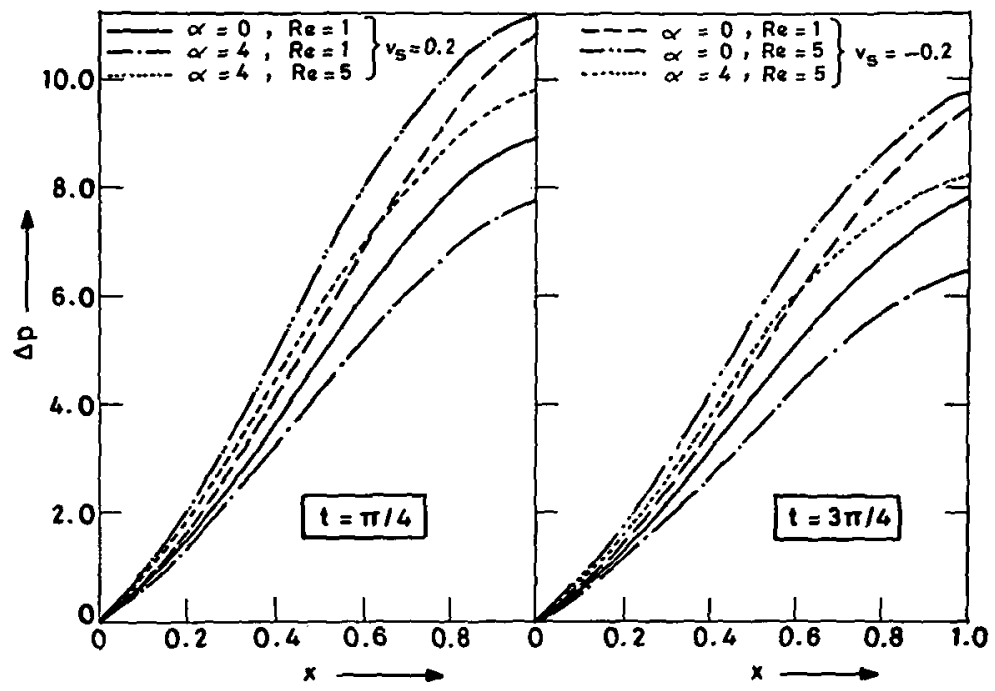

FIGURE 7. $\Delta p$ vs $x$ for locally constricted tube.

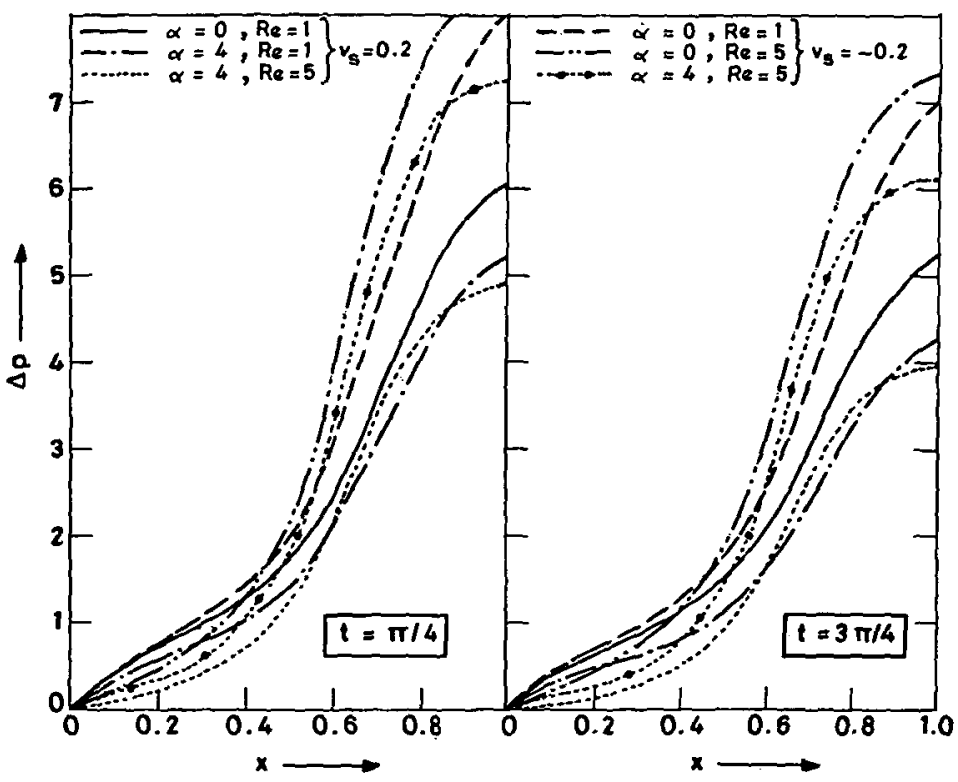

FIGURE 8. $\Delta p$ vs $x$ for sinusoidal tube. 


\section{Conclusion}

Here we have studied the effect of suction/injection on pulsatile flow through circular tubes of varying cross-section. It is observed that the effect of suction on the wall of the tube is to decrease shear stress at the wall as well as the pressure drop. The wall shear stress shows periodic behaviour with time and it decreases as the frequency parameter increases. Further, an increase in the Reynolds number results in an increase of the wall shear stress in the converging portion and in its decrease in the diverging portions of the tube.

\section{Acknowledgement}

The authors are thankful to the referee for his constructive remarks to improve the presentation of the paper.

\section{References}

[1] J. P. Bitoun and D. Bellet, "Blood flow through a stenosis in microcirculation", Biorheology 23 (1986) 51-61.

[2] P. A. Gaeghtgens, "Pulsatile pressure and flow in the mesentric vascular bed of the cat", Pflugers Arch. 316 (1970) 14-15.

[3] R. I. Macey, "Pressure flow patterns in a cylinder with reabsorbing walls", Bull. Math. Biophys. 25 (1963) 1-9.

[4] R. I. Macey, "Hydrodynamics in renal tubules", Bull. Math. Biophys. 27 (1965) 117-124.

[5] M. J. Manton, "Low reynolds number flow in slowly varying axisymmetric tubes", J. Fluid Mech. 49 (1971) 451-459.

[6] G. Radhakrishnamacharya, Peeyush Chandra and M. R. Kaimal, "A hydrodynamical study of flow in renal tubule", Bull. Math. Biol. 43 (1981) 151-163.

[7] A. Ramachandra Rao and Rathna Devanathan, "Pulsatile flow in tubes of varying crosssection", Z. A. M. P. 24 (1973) 203-213.

[8] D. J. Schneck and S. Ostrach, "Pulsatile blood flow in a channel of small exponential divergence-I. The linear approximation for low mean Reynolds number”, J. Fluids Eng. 16 (1975) 353-360.

[9] C. H. Weiderheilm, J. W. Woodbury, S. Kink and R. F. Rushmer, "Pulsatile pressures in the microcirculation of frog's mesentry", Amer. J. Physiol. 207 (1969) 173-176.

[10] J. R. Womersley, "Method for calculation of velocity, rate of flow and viscous drag when pressure gradient is known”, J. Physiol. 127 (1955) 553-563.

[11] J. R. Womersley, "Oscillatory motion of a viscous liquid in a thin walled elastic tube. I. The linear approximation for long waves", Phil. Mag. 46 (1955) 199-221. 